\title{
PRODUCTION OF HALO PARTICLES BY COLLECTIVE MODE EXCITATIONS IN HIGH INTENSITY CHARGED PARTICLE BEAMS
}

\author{
Sean Strasburg and Ronald C. Davidson, Princeton Plasma Physics Laboratory
}

\section{Abstract}

This paper examines the effects of self-consistent collective oscillations excited in a high-intensity ion beam on the motion of a test particle in the beam core. Even under ideal conditions, assuming a constant transverse focusing force (smooth focusing approximation), and perturbations about a uniform-density, constant-radius beam, it is found that collective mode excitations, in combination with the applied focusing force and the equilibrium self fields, can eject particles from the beam core to large radii.

\section{INTRODUCTION}

It is increasingly important to develop improved theoretical models of halo production and control for chargedparticle beam propagation in high-intensity accelerators and transport systems[1], with applications to spallation neutron sources, heavy ion fusion, nuclear waste treatment, and tritium production. While halo formation mechanisms, such as beam mismatch and nonlinearities associated with nonuniform space-charge forces have been explored both analytically and numerically[2, 3], a fundamental understanding of halo production is incomplete. In this paper, we consider a new mechanism for the production of halo particles. Namely, we consider, for the first time, the effects of self-consistent collective oscillations excited in a high-intensity ion beam on the motion of a test particle in the beam core. Even under ideal conditions, assuming a constant transverse focusing force (smooth focusing approximation), and perturbations about a uniform-density, constant-radius beam, it is found that collective mode excitations, in combination with the applied focusing force and the equilibrium self fields, can eject particles from the beam core to large radii.

\section{THEORETICAL MODEL AND ASSUMPTIONS}

We consider an intense nonneutral ion beam with characteristic beam radius $R$ and axial momentum $\gamma_{b} m \beta_{b} c$ propagating in the $z$-direction with average axial velocity $V_{b}=\beta_{b} c=$ onst .. The applied transverse focusing force in the smooth focusing approximation is modeled by $\mathbf{F}_{f_{0}}(\mathbf{x})=-\gamma_{b} m \omega_{f}^{2}\left(x^{\wedge} \mathbf{e}+\hat{y^{2}} \mathbf{g}\right)$. The effects of selfelectric and self-magnetic fields on the particle dynamics are retained in a self-consistent manner, consistent with the paraxial approximation, and the assumption that Budker's parameter satisifes $\nu_{B}=N_{b}(Z e)^{2} / m c^{2} \ll \gamma_{b}$. Here, $N_{b}$ is the number of beam ions per unit axial length, related to the number density of beam ions $n_{b}(x y, s)$ by $N_{b}=$ $\int d x d y b$, where $s=\beta_{b} c t$. The wavenumber equivalent to the transverse focusing frequency, the transverse focusing coefficient $\kappa$, is defined by $\sqrt{\kappa}=\omega_{f} / \beta_{b} c$, which has units of inverse length. We further assume axisymmetric unbunched beam propagation $(\partial / \partial \theta=0=\partial / \partial z)$, and introduce the normalized dimensionless self-field potential $\psi$ defined by $\psi(r, s)=Z e \phi(r, s) / \gamma_{b}^{3} m \beta_{b}^{2} c^{2}$.

We assume a kinetic or warm-fluid[4] KapchinksijVladimirskij (KV) beam equilibrium, and for this case the equilibrium density profile $n_{b}^{0}(r, s)$ has the uniform value $N_{b} / \pi R^{2}$ in the beam interior and is equal to zero outside the beam, defined by $r>R(s)$, where $R(s)$ is the solution to the envelope equation[2]. We further assume a matched, constant-radius beam equilibrium with $R(s)=R_{0}$, a constant, given by $\left(\kappa-K / R_{0}^{2}\right) R_{0}=\epsilon^{2} / R_{0}^{3}$. Here $\epsilon$ is the unnormalized transverse emittance, and $K$ is the selffield perveance defined by $K=2 N_{b}(Z e)^{2} / \gamma_{b}^{3} m\left(\beta_{b} c\right)^{2}$. The "depressed" oscillation wavenumber $\bar{\nu}$ (dimensionless units) is defined in terms of the transverse focusing coefficient $\kappa$, perveance $K$, and equilibrium beam radius $R_{0}$, by $\bar{\nu}^{2}=1-K / \kappa R_{0}^{2}$. The "depressed" oscillation wavenumber $\omega$ (dimensional units) is given by $\omega^{2}=\kappa \bar{\nu}^{2}$.

A key focus of the present analysis is to investigate the motion of a test ion in the combined force of the applied focusing field $\mathbf{F}_{\text {fo }}$, the equilibrium self fields, and the perturbed self fields associated with self-consistent collective oscillations excited in the beam. We express the total selffield potential as $\psi(\bar{r}, s)=\psi_{0}(\bar{r})+\delta \psi(\bar{r}, s)$, where $\psi_{0}(\bar{r})$ is produced by the step-function equilibrium density profile and $\bar{r}=r / R_{0}$ is the normalized radial coordinate. For the perturbed potential $\delta \psi(\bar{r}, s)$, we make use of the warm-fluid model developed by Lund and Davidson[4]. This model, simplified by the assumptions of cylindrical symmetry, predicts an infinite class of collective modes $\delta \psi_{n}$, vanishing outside the beam core, with purely radial dependence, and stably oscillating with eigenfrequency $\omega_{n}$. The radial eigenfunction $\delta \psi_{n}(\bar{r})$ in the beam interior $(0 \leq \bar{r}<1)$ is defined in terms of the Legendre polynomials (of the first kind), $P_{n-1}(x)$ and $P_{n}(x)$, by $\delta \psi_{n}(\bar{r})=\frac{1}{2} A_{n}\left[P_{n-1}\left(1-2 \bar{r}^{2}\right)+P_{n}\left(1-2 \bar{r}^{2}\right)\right]$, where $\left\{A_{n}\right\}$ are constant amplitudes. The normal-mode oscillation wavenumbers $\left\{\omega_{n}\right\}$ are defined in Ref. [4] by $\omega_{n}^{2}=$ $\kappa\left[2+2^{-} \nu^{2}\left(2 n^{2}-1\right)\right]$.

It is readily shown that the equation of motion in the applied and equilibrium self fields, together with the oscillating collective modes, is given by

$$
\frac{d^{2} \bar{r}}{d s^{2}}+\left(\kappa+\frac{2}{R_{0}^{2}} \frac{\partial \psi}{\partial \bar{r}^{2}}\right) \bar{r}=\frac{\bar{P}_{\theta}^{2}}{\bar{r}^{3}}
$$

where $\bar{P}_{\theta}$ is the (normalized) canonical angular momentum, $R_{0}^{2} \bar{P}_{\theta}=x y^{\prime}-y x^{\prime}$, which is a constant of the motion. Here $\psi(\bar{r}, s)=\psi_{0}(\bar{r})+\delta \psi(\bar{r}, s)$, where the eigenfunctions 
are the Legendre polynomials defined above and the eigenfrequencies are $\left\{\omega_{n}\right\}$. Equation (1) is a valid description of the test ion motion, both inside the beam $(\bar{r}<1)$ and outside the beam $(\bar{r}>1)$.

For test-particle motion inside the beam, we obtain[6]

$$
\frac{d^{2} \bar{r}}{d s^{2}}+\omega^{2} \bar{r}-\frac{\bar{P}_{\theta}^{2}}{\bar{r}^{3}}=\frac{K}{R_{0}^{2}} \sum_{n=1}^{\infty} A_{n} \delta \psi_{n}^{\prime}(\bar{r}) \cos \omega_{n} s .
$$

On the other hand, for particle motion outside the beam, Eq. (1) reduces to the nonlinear autonomous equation

$$
\frac{d^{2} \bar{r}}{d s^{2}}+\left(\kappa-\frac{K}{R_{0}^{2} \bar{r}^{2}}\right) \bar{r}-\frac{\bar{P}_{\theta}^{2}}{\bar{r}^{3}}=0 .
$$

For case of vanishing angular momentum (meridional particles), setting $\bar{P}_{\theta}=0$ in Eqs. (2) and (3) and replacing $\bar{r}$ with $\bar{x}=x / R_{0}$ yields the appropriate equations.

Equations (2) and (3), supplemented by the associated definitions of $\delta \psi_{n},\left\{\omega_{n}\right\}$, etc., constitute the final forms of the test-particle orbit equations to be investigated analytically and numerically in Secs. 3 and 4.

\section{THEORY OF THE DYNAMICAL SYSTEMS}

In this section we examine several features of the test ion motion analytically, using numerical solutions as verification.

\subsection{Resonant Behaviour}

Meridional Test Ions We begin by considering meridional particles with $\bar{P}_{\theta}=0$ in a beam supporting a single collective mode with eigennumber $n$. The functional relationship between the $n^{\text {th }}$ mode frequency $\omega_{n}$ and the depressed transverse oscillation frequency $\omega$ makes fundamental and principal resonances "inaccessible" for all mode numbers $n$. The Hamiltonian expansions predict resonances for the $n^{\text {th }}$ mode at $\omega_{n} / \omega= \pm 2 m$ for integers $m \leq n$. Using $\left\{\omega_{n}\right\}$, we can easily obtain an expression for $\omega_{n}^{2} / \omega^{2} \equiv g_{n}^{2}$. The minimum of $g_{n}$ occurs at $\bar{\nu}^{2}=1$ at which $g_{n}=2 n$, the uppermost resonance. However, as this resonance is approached, the coefficient which multiplies the mode amplitude, $K / R_{0}^{2}=\kappa\left(1-\bar{\nu}^{2}\right)$, approaches zero.

Equation (2) for meridional particles inside a beam supporting an $n=1$ mode is a Mathieu equation, the same as that generated by an envelope oscillation $R(s)=R_{0}(1+$ $\left.\frac{1}{2} \delta_{e} \cos \omega_{e} s\right)$ in the limit of small $\delta_{e}$, taking $\delta_{e}=\delta_{1}$ and $\omega_{e}=\omega_{1}$. However, it is important to distinguish what constitutes inside the beam for the two cases. The fluid modes are derived for perturbations about a constant radius beam, so inside the beam corresponds to $|\bar{x}|<1$, or $|x|<R_{0}$. The mismatch ripple, however, requires $|x|<R(s)=R_{0}\left(1+\frac{1}{2} \delta_{e} \cos \omega_{e} s\right)$. Since $R(s)$ is the projection of an energy level, particles initially inside the energy shell of the beam will remain confined in the interior of a mismatched beam for all time, whereas the beam edge $R_{0}$ of a beam with an $n=1$ mode is not a node of the perturbed potential, and so particles may escape.

Test Ions With Nonzero Angular Momentum As a simple example for particles with nonzero angular momentum $\bar{P}_{\theta} \neq 0$, we consider perturbations around an interior circular orbit with constant radius $\bar{r}_{c}=r_{c} / R_{0}$, requiring that $\bar{P}_{\theta}^{2}=\bar{r}_{c}^{4} \omega^{2}$. The frequency of small radial oscillations about the equilibrium orbit is found to be $\omega_{c}=2 \omega$, independent of the equilibrium radius $\bar{r}_{c}$. If these orbit oscillations resonate with the collective mode, the particle will experience a significant energy change.

The linearization for small radial oscillations has fundamental and principal resonances when the ratio $\omega_{1} / \omega_{c}=$ $1,2 m$ for integers $m \leq n$. For $n=1$, the fundamental resonance is at $\bar{\nu}^{2}=1$, and the Mathieu (or principal) resonance is at $\bar{\nu}^{2}=\frac{1}{7}$. Both have important effects on the dynamics of a single particle.

\subsection{Non-Resonant Behaviour}

It is also possible for a test particle to nonresonantly gain enough energy in the beam interior to escape the beam, disrupting the process of giving the energy back. All particles with sufficient energy in the unperturbed case to attain a radius greater than $\bar{x}_{\Delta}<1$ will be ejected by the collective modes into the highly nonlinear region exterior to the beam at some point of their trajectory. This minimum expelled radius $\bar{x}_{\Delta}$ is a strong function of the mode amplitude strength $\Gamma$, defined as the rms field energy in the $n^{\text {th }}$ mode divided by the rms electrostatic energy in the beam core. The function $\bar{x}_{\Delta}$ may be calculated for low $n$ by transforming the perturbation in the Hamiltonian to higherorder[5], yielding a new energy-like invariant, the new action $\tilde{J}$, whose maximum is directly related to the maximum excursion of the perturbed particle trajectory. For $n=2$, the unperturbed maximal radius which will just reach the beam edge due to the collective mode is given by[6]

$$
\bar{x}_{\Delta}^{2}=\frac{-\left(1+\epsilon c_{2}\right) \pm\left[\left(1+\epsilon c_{2}\right)^{2}+2 \epsilon c_{1} \omega\right]^{1 / 2}}{\epsilon \omega c_{1}} .
$$

The values $c_{1}$ and $c_{2}$ depend only on the depressed transverse frequency and the ratio $\alpha \equiv \omega_{2} / \omega$, which in turn depend only on $\bar{\nu}$, and are given by $c_{1}=c_{3}\left(21 \alpha-\frac{3}{4} \alpha^{3}\right)$ and $c_{2}=c_{3} \omega\left(-16 \alpha+\alpha^{3}\right)$, where $c_{3}^{-1}=\omega^{3}\left(64-20 \alpha^{2}+\alpha^{4}\right)$. Here, we define $\epsilon=\left(K / R_{0}\right) \sqrt{\Gamma}$. This expression gives, within a few percent over a wide range of parameters, the extent of the region near the beam edge which will be ejected from the beam at some point of the trajectory. Figure 1 shows $\bar{x}_{\Delta}$ as a function of normalized mode energy $\Gamma$ in a range over which it is an accurate approximation.

\section{NUMERICAL RESULTS}

The dynamics become more complicated when a trajectory spends time both inside and outside the beam, and numerical solutions of the equations are easiest under these conditions. 


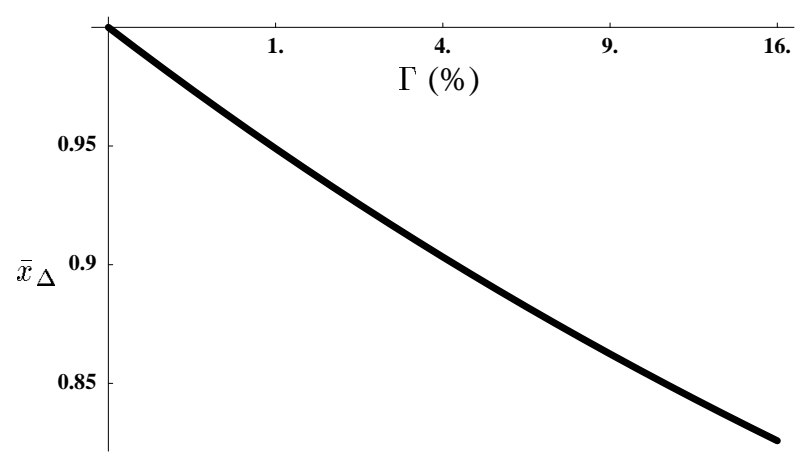

Figure 1: Plot of $\bar{x}_{\Delta}$ versus $\Gamma(\%)$ for $\bar{\nu}^{2}=\frac{1}{4}$.

Particles whose energy corresponds to a maximum unperturbed trajectory, which we denote $\bar{x}_{0}$, between $\bar{x}_{\Delta}$ and 1 will be ejected from the beam at some point, with the possibility of large energy gains. In general, ejected particles either $(i)$ experience negligible energy gains, or (ii) obtain a well-defined maximum excursion $\bar{X}_{1} \simeq 1$.5. This behaviour is a function of space-charge depression $\bar{\nu}, \mathrm{rms}$ field energy in the $n^{\text {th }}$ mode relative to rms electrostatic energy in the beam core $\Gamma \equiv \mathcal{E}_{n} / \mathcal{E}_{0}$, and the ejected particle's unperturbed maximum radius $\bar{x}_{0}$.

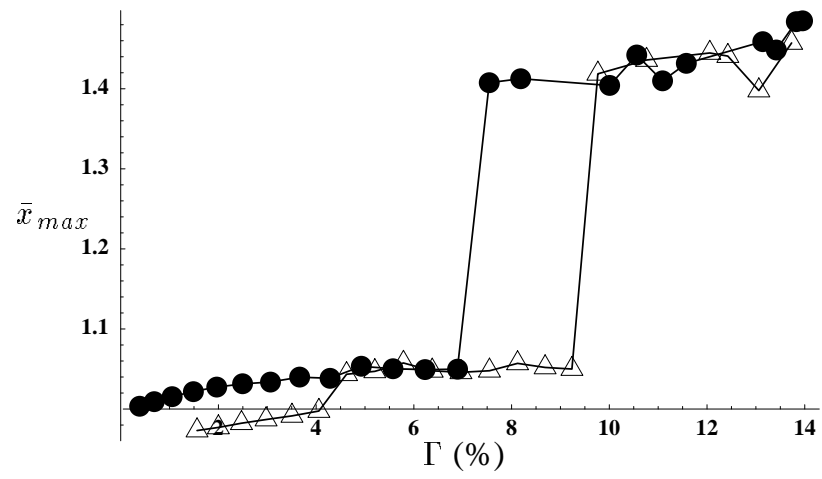

Figure 2: Plot of $\bar{x}_{\max }$ versus $\Gamma(\%)$ for $\bar{\nu}^{2}=\frac{1}{3}$ with $\bar{x}_{0}=$ $0.99(\bullet)$ and $\bar{x}_{0}=0.95(\triangle)$.

For mode energies less than a certain critical energy $\Gamma_{1}\left(\bar{\nu}, \bar{x}_{0}\right)$, particles go no further than a few percent of $R_{0}$ outside of the beam; for energies greater than $\Gamma_{1}$, particles consistently travel as far out as $\bar{X}_{1} \simeq 1.5$ (see Fig. 2 ). The value of $\Gamma_{1}$ decreases as the beam becomes more intense, ranging from less than $2 \%$ at $\bar{\nu}^{2}=\frac{1}{5}$ to $15 \%$ at $\bar{\nu}^{2}=\frac{1}{2}$. In addition, particles with $\bar{x}_{0}$ further from the beam edge have slightly higher critical energies, and of course particles with $\bar{x}_{0}<\bar{x}_{\Delta}$ never leave the beam. The value of $\bar{X}_{1}$ gradually increases with $\Gamma$, and depends weakly on $\bar{\nu}$. Since $\bar{X}_{1}$ does not depend on $\bar{x}_{0}$, this is the largest radius any particle initially in the beam can escape to, and functions as a KAM curve, giving an indication of the phase space structure in the halo region.

Finally, for intense beams with sufficiently large amplitude modes, this phase-space spanning curve can be destabilized and break into islands. Above a critical energy $\Gamma_{2}$,

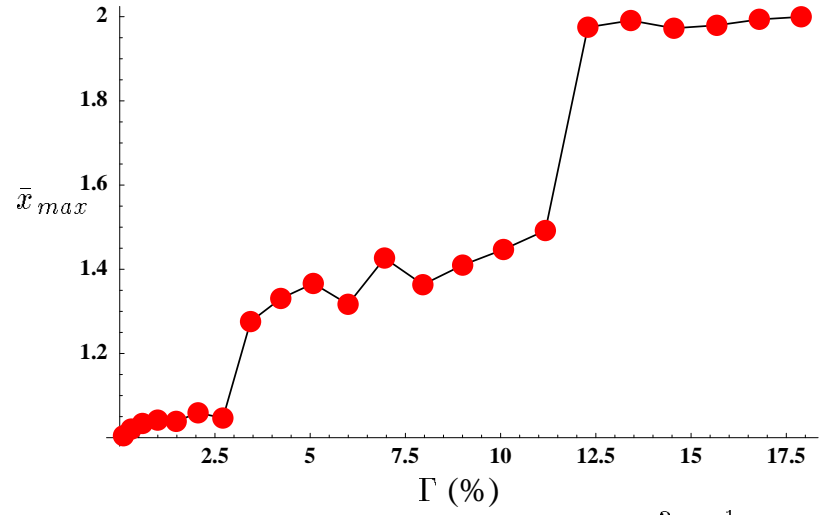

Figure 3: Plot of $\bar{x}_{\max }$ versus $\Gamma(\%)$ for $\bar{\nu}^{2}=\frac{1}{5}$.

particles can explore out to $\bar{X}_{2} \simeq 2$ (see Fig. 3 ). It is plausible that, for extremely intense beams, larger collective mode amplitudes would make accessible even greater regions of phase space.

\section{CONCLUSIONS}

We have explored the range of particles capable of being expelled from the beam core by collective mode excitations, and the maximum radii they can attain as KAM surfaces are successively destabilized with increasing perturbation strength. These processes occur under even ideal conditions, assuming constant transverse focusing force and a uniform-density matched-beam equilibrium.

\section{ACKNOWLEDGEMENTS}

This research was supported by the Department of Energy and the Department of Defense.

\section{REFERENCES}

[1] R. C. Davidson, Physics of Nonneutral Plasmas (AddisonWesley Publishing Co., Reading, MA, 1990); M Reiser, Theory and Design of Charged-Particle Beams (Wiley, NY, 1994); and references therein.

[2] Space Charge Dominated Beams and Applications of High Brightness Beams, ed. S. Y. Lee, AIP Conf. Proc. 377 (AIP, NY, 1996), and references therein.

[3] R. Gluckstern et al., Phys.Rev.Lett. 75, 2835 (1995); R. Gluckstern, Phys.Rev.Lett. 73, 1247 (1994). A. Riabko et al., Phys.Rev. E 51, 3529 (1995).

[4] S. Lund and R. C. Davidson, Phys. Plasmas 5, 3028 (1998).

[5] A. J. Lichtenberg and M. A. Lieberman, Regular and Chaotic Dynamics, 2nd ed. (Springer-Verlag, NY, 1992), and refereces therein.

[6] S. Strasburg and R. C. Davidson, to be published. 